\title{
Clinical Relevance of As-Needed Treatment with Nalmefene in Alcohol-Dependent Patients
}

\author{
Henri-Jean Aubin ${ }^{a}$ Jens Reimer ${ }^{b}$ David J. Nutt ${ }^{c}$ Anna Bladström ${ }^{d}$ \\ Lars Torup $^{d}$ Clément François $^{\mathrm{e}}$ Jonathan Chick $^{f}$

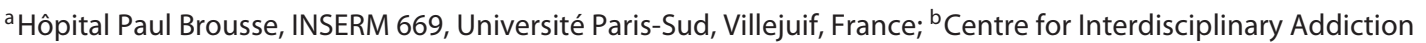 \\ Research of Hamburg University, Hamburg, Germany; ' ${ }^{\complement}$ Department of Medicine, Imperial College, London, \\ United Kingdom; ${ }^{d} \mathrm{H}$. Lundbeck A/S, Valby, Denmark; ${ }^{\mathrm{e}} \mathrm{H}$. Lundbeck A/S, Issy les Moulineaux cedex, France; \\ ${ }^{f}$ Health Sciences, Queen Margaret University, Edinburgh, United Kingdom
}

\section{Key Words}

Alcohol dependence - Harm reduction - Nalmefene .

Opioid antagonist $\cdot$ Clinical relevance

\begin{abstract}
Nalmefene is the first drug approved for reduction of alcohol consumption. The aim of this study was to evaluate the clinical relevance of treatment with nalmefene in alcohol-dependent patients with a high drinking risk level from two randomised placebo-controlled 6-month studies (NCT00811720 and NCT00812461). Response criteria were based on alcohol consumption, Clinical Global Impression, and Short Form Health Survey mental component summary scores at month 6, analysed using logistic regression. The proportion of responders was higher in the nalmefene group than in the placebo group with odds ratios significantly in favour of nalmefene for all responder criteria; numbers-needed-to-treat ranged from 6 to 10. Significant differences from placebo in clinician-rated and patient-reported outcomes, and liver enzymes further supported the clinical relevance of the treatment effect. In conclusion, this study supports the clinical relevance of nalmefene treatment in patients with alcohol dependence. Nalmefene may help to reduce the alcohol-related burden and the large treatment gap, with currently less than $10 \%$ of alcohol-dependent patients in Europe receiving treatment.
\end{abstract}

(c) 2015 S. Karger AG, Basel

\begin{tabular}{ll}
\hline KARGER 125/s & $\begin{array}{l}\text { ( } 2015 \text { S. Karger AG, Basel } \\
\text { 1022-6877/15/0213-0160\$39.50/0 }\end{array}$ \\
$\begin{array}{l}\text { E-Mail karger@karger.com } \\
\text { www.karger.com/ear }\end{array}$ & $\begin{array}{l}\text { This is an Open Access article licensed under the terms of the } \\
\text { Creative Commons Attribution-NonCommercial 3.0 Un- } \\
\text { ported license (CC BY-NC) (www.karger.com/OA-license), } \\
\text { applicable to the online version of the article only. Distribu- } \\
\text { tion permitted for non-commercial purposes only. }\end{array}$
\end{tabular}

\section{Introduction}

Nalmefene is an opioid system modulator, with antagonist activity at the $\mu$ - and $\delta$-receptors and partial agonist activity at the $\kappa$-receptor [1-4]. It was recently granted market authorisation in the European Union as the first pharmacological therapy approved for the reduction of alcohol consumption in adult patients with alcohol dependence that have a high drinking risk level according to the World Health Organisation [5] (men: >60 g/day and women: $>40 \mathrm{~g} /$ day) and who continue to have that 2 weeks after initial assessment [6].

What constitutes a clinically relevant magnitude of reduction of alcohol consumption? Strictly speaking, clinical significance is defined as returning to normal functioning [7]. However, this criterion is often viewed as too stringent, and therefore clinical significance usually refers to quantified information about the importance of the finding, using metrics such as effect size or number-needed-to-treat. The use of responder categories to express the clinical relevance of a treatment has been argued to provide more clinically relevant information than mean differences on severity scales [8].

\section{H.-J.A. and J.R. contributed equally to this work.}

Professor Henri-Jean Aubin, MD

Centre d'Enseignement, de Recherche et de Traitement des Addictions Hôpital Paul Brousse, AP-HP, Université Paris-Sud INSERM U669, FR-94804 Villejuif (France)

E-Mail henri-jean.aubin@ @br.aphp.fr 
Increasing attention has been focused recently on the importance of patient-reported outcomes, e.g. quality of life, in informing research results and clinical practice [9]. Similarly, Clinical Global Impression (CGI) measurements allow a broader assessment of patients' improvement than primary endpoints alone. Change to baseline of validated liver biomarkers offer an objective confirmation of the findings based on patients' reports.

The measure of the clinical relevance of a drug also refers to a comparison with competing treatment options. As nalmefene is the first drug labelled for alcohol reduction in alcohol-dependent subjects, the comparison of effect size would by necessity be made with drugs labelled for abstinence maintenance, or more generally with psychiatric medications.

This article describes an evaluation of the clinical relevance of the reduction of alcohol consumption by nalmefene treatment in patients with high or very high drinking risk levels as recently described by van den Brink et al. [10].

\section{Methods}

\section{Patients}

This paper includes data from the pooled sub-group of patients with a high or very high drinking risk level at screening and randomisation (target population as defined in [10]) from the identically designed 6-month studies ESENSE1 (NCT00811720) [2] and ESENSE2 (NCT00812461) [3]. Briefly, the studies included adult patients with a primary diagnosis of alcohol dependence according to the Diagnostic and Statistical Manual of Mental Disorders [11], assessed with the Mini-International Neuropsychiatric Interview [12]. Patients were excluded if they had comorbid psychiatric disorders or an average alcohol consumption below a medium drinking risk level (men: $\leq 40 \mathrm{~g}$ alcohol/day; women $\leq 20 \mathrm{~g}$ alcohol/day) according to the World Health Organization [5]. The full list of selection criteria is published elsewhere $[2,3]$. Both studies were designed and conducted in accordance with the principles of the Declaration of Helsinki and Good Clinical Practice, and each site started patient inclusion only after ethics committee approval. All patients gave written informed consent.

\section{Study Procedures}

Patients were assigned to 24 weeks of double-blind treatment with as-needed use of either placebo or nalmefene in a 1:1 ratio (see $[2,3]$ for details). Patients were instructed to take one tablet on each day they perceived a risk of drinking alcohol ('as-needed dosing'), preferably $1-2 \mathrm{~h}$ prior to the anticipated time of drinking, but otherwise as soon after drinking had started. In addition, all patients took part in a low-intensity motivational and adherence-enhancing intervention (BRENDA) [13] starting at randomisation and subsequently at all scheduled visits. No treatment goal was defined, i.e. both abstinence and reduced-risk drinking were accepted, and no information was collected on individual treatment goals.

As-Needed Treatment with Nalmefene in

Alcohol-Dependent Patients
Assessments of efficacy and safety were performed at screening, randomisation, and weeks 1,2, and 4, followed by monthly assessments. Monthly drinking variables were derived from the Time Line Follow-Back used to provide information of daily number of standard drinks [14]. At screening, patients reported their daily drinking over the previous month (i.e. 28 consecutive days). At subsequent visits, they reported on their drinking since the previous visit. For the full list of study procedures, see Mann et al. [2] and Gual et al. [3].

The protocol pre-specified co-primary outcome measures were change from baseline in the monthly number of heavy drinking days (HDDs) and total alcohol consumption in grams of pure alcohol per day at month 6 . The clinical relevance of the reduction in HDDs and total alcohol consumption described in van den Brink et al. [10] was studied using the Clinical Global Impression Severity of Illness and Improvement scales (CGI-S and CGI-I) [15], Short Form Health Survey version 2 (SF-36) [16] mental component summary (MCS) and physical component summary (PCS) scores, Drinker Inventory of Consequences (DrInC-2R) [17], Alcohol Dependence Scale (ADS) [18], and serum levels of the liver enzymes $\gamma$-glutamyltransferase (GGT), alanine aminotransferase (ALAT), and aspartate aminotransferase (ASAT), as markers of hepatic cytolysis, liver disorder being an important clinical outcome in such patients.

Clinical relevance was also investigated using various definitions of response at month 6 (responder criteria based on alcohol consumption)/week 24 (responder criteria based on CGI-S, CGI-I, and SF-36 MCS score):

- Two-category downward shift from baseline in drinking risk level

- Shift to low drinking risk level

- 2-point downward shift in CGI-S compared to baseline

- Shift to CGI-S $\leq 3$ (mildly ill, borderline ill, or normal)

- Shift to CGI-I $\leq 2$ (much improved or very much improved)

- $\quad$ Shift to SF-36 MCS score $>47$

The WHO-defined (WHO, 2000) drinking risk levels are as follows:

- Very high risk - men: $>100 \mathrm{~g} /$ day, women: $60 \mathrm{~g} /$ day

- High risk - men: $>60$ to $100 \mathrm{~g} /$ day, women: $>40$ to $60 \mathrm{~g} / \mathrm{day}$

- Medium risk - men: $>40$ to $60 \mathrm{~g} /$ day, women: $>20$ to $40 \mathrm{~g} /$ day

- Low risk - men: 1-40 g/day, women: 1-20 g/day

The rationale for choosing shift to SF-36 MCS score $>47$ was that 50 is the MCS score of the general population, and following the definition of a minimal clinically important difference [19] equal to 3, then 47 (50 minus the minimal clinically important difference) would represent the lowest value for considering an individual being representative of the general population.

\section{Statistical Analyses}

Demographics and baseline clinical characteristics are presented for the target population. Efficacy analyses were based on patients in the target efficacy population (all patients in the target population with recorded study medication intake and $\geq 1$ valid post-baseline assessment of HDDs and total alcohol consumption) as defined in van den Brink et al. [10]. In the analyses, it was also required that each variable had a baseline assessment for the actual scale.

The baseline for drinking variables was defined as the month preceding the screening visit. For all other variables, the baseline 
was defined as the assessment at the screening visit. ADS was only measured at baseline and week 24 and was therefore analysed by analysis of covariance (ANCOVA), using baseline observation carried forward for missing values, with baseline score as a covariate and country, sex, and treatment as factors. The other continuous outcomes were analysed using a mixed model repeated measures which compensates for missing values, with an unstructured covariance matrix, using available data, with the baseline score as the covariate (for CGI-I, CGI-S score at baseline was used), and country, sex, time, and treatment as fixed effects; baseline score-by-time interaction and treatment-by-time interaction were also included in the model. The Kenward-Roger approximation was used to estimate denominator degrees of freedom. CGI-S, SF-36 MCS score, SF-36 PCS score, ADS total score, and DrInC-2R total score were analysed using change from baseline variables. GGT, ALAT, and ASAT were analysed using log-transformed values; the adjusted means were back-transformed using the exponential function to obtain the geometric means.

Responder endpoints were analysed using logistic regression, with country, sex, baseline drinking risk level or baseline score (SF36 mental component grouped in 10-point categories and CGI-S for CGI-S and CGI-I), and treatment as factors. Missing values were imputed with response based on values predicted from the mixed model repeated measures. Robustness of the results was investigated by imputing missing values as: non-response for the shift to less than or equal to low and two-category downshift in drinking risk level, and worst observation including all available observations for the patients for response based on CGI and SF-36 MCS score. Odds ratios are presented with two-sided 95\% CI and corresponding $\mathrm{p}$ values based on likelihood ratio tests.

For shift to less than or equal to low and two-category downward shift in drinking risk level, the number of HDDs at month 6 was tabulated by treatment group for patients achieving response at month 6 . Patients with $>12$ HDDs were grouped.

All statistical tests were two-sided; $p$ values $<0.05$ were referred to as being statistically significant. The statistical software used was $\mathrm{SAS}^{\circledR}$, version 9.2 .

\section{Results}

\section{Study Sample}

The target population comprised 667 patients (placebo: 332; nalmefene: 335; fig. 1), and the target efficacy population comprised 641 patients (placebo: 322; nalmefene: 319).

As previously described [10], patients were predominantly middle-aged men, with more than 10 years of drinking problems and no previous treatment for alcohol dependence. Further, approximately $60 \%$ of the patients had a very high drinking risk level and the average patient had a SF-36 MCS score of approximately 40, which is below the average for a general population (table 1).

There were no differences in demographics, alcohol history, or other baseline values between the nalmefene and placebo group.

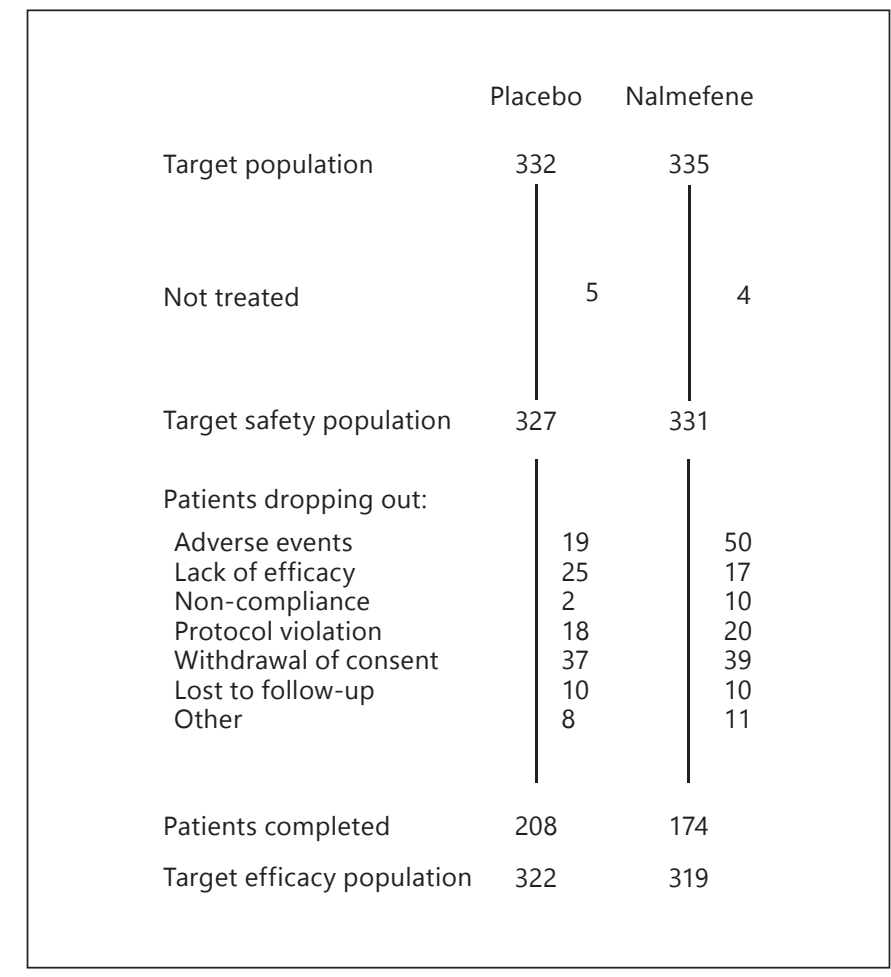

Fig. 1. Trial profile.

\section{Responder Analyses}

The proportion of responders at month 6/week 24 was higher in the nalmefene group than in the placebo group with odds ratios for response consistently significantly in favour of nalmefene for all defined responder criteria (fig. 2; table 2).

The proportions of responders translated to numbers-needed-to-treat ranged from 6 to 10 (table 2). The sensitivity analyses supported the primary analyses, although 2 out of 6 did not reach statistical significance (supporting information in online suppl. table S1; for all online suppl. material, see www.karger.com/ doi/10.1159/000371547).

\section{Distribution of HDDs at Month 6}

The distribution of HDDs for patients defined as responders based on a two-category downshift and shift to low drinking risk level is presented in figure 3. Approximately a third of these patients had no HDDs at month 6 .

\section{Clinician-Rated Scales}

The clinical relevance of the reductions in HDD and total alcohol consumption was also shown by the improvements in the CGI scores (fig. 4). 
Table 1. Demographics and baseline clinical characteristics

\begin{tabular}{|c|c|c|}
\hline Race (Caucasian) & $329(99.1 \%)$ & $333(99.4 \%)$ \\
\hline Sex (men) & $216(65.1 \%)$ & $223(66.6 \%)$ \\
\hline Age, years & $48.7(10.5)$ & $48.4(10.5)$ \\
\hline $\mathrm{BMI}, \mathrm{kg} / \mathrm{m}^{2}$ & $26.1(4.4)$ & $26.0(4.8)$ \\
\hline Age at onset of drinking problem, years & $35.1(11.6)$ & $35.6(12.3)$ \\
\hline \multicolumn{3}{|l|}{ Drinking risk level - WHO classification ${ }^{\mathrm{a}}$} \\
\hline High & $139(41.9 \%)$ & $124(37.0 \%)$ \\
\hline Very high & $193(58.1 \%)$ & $211(63.0 \%)$ \\
\hline CGI-S & $4.3(1.4)$ & $4.3(1.4)$ \\
\hline Carbohydrate-deficient transferrin, \% & $2.6(1.5)$ & $2.8(1.7)$ \\
\hline DrInC-2R total score & $42.2(22.2)$ & $41.1(22.3)$ \\
\hline ADS total score & $13.3(5.7)$ & $14.0(6.0)$ \\
\hline SF-36 PCS score & $50.1(8.0)$ & $50.8(8.0)$ \\
\hline SF-36 MCS score & $40.7(12.6)$ & $40.9(11.9)$ \\
\hline Living with someone (yes) & $233(70.2 \%)$ & $247(73.7 \%)$ \\
\hline Active employment (yes) & $183(55.1 \%)$ & $197(58.8 \%)$ \\
\hline Higher education (yes) & $96(28.9 \%)$ & $98(29.3 \%)$ \\
\hline Previously treated for alcohol dependence (yes) & $112(33.7 \%)$ & $105(31.3 \%)$ \\
\hline
\end{tabular}

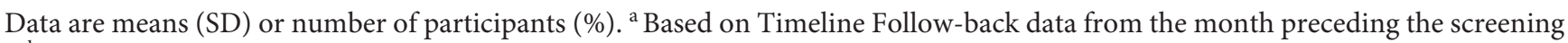
visit. ${ }^{\mathrm{b}}$ Geometric mean.

At baseline, the mean CGI-S score was 4.3 for both placebo and nalmefene. The adjusted mean change in the CGI-S score [standard error (SE)] at week 24 was -0.8 (0.1) for placebo and -1.3 (0.1) for nalmefene, corresponding to a treatment effect of -0.5 ( $95 \% \mathrm{CI}:-0.7$ to $-0.2, \mathrm{p}<$ 0.0001 ) in favour of nalmefene. The adjusted mean CGI-I score (SE) at month 6 was 2.9 (0.1) for placebo and 2.5 (0.1) for nalmefene, resulting in a significant difference from placebo of -0.4 ( $95 \% \mathrm{CI}:-0.6$ to $-0.2, \mathrm{p}<0.0001$ ).

At baseline, the mean ADS score was 13.3 in the placebo group and 14.1 in the nalmefene group. At week 24, the estimated mean change from baseline in ADS score (SE) was -3.5 (0.4) for the placebo group and -5.1 (0.4) for the nalmefene group, corresponding to a treatment difference of -1.6 (95\% CI: -2.4 to $-0.8, \mathrm{p}<0.0001)$ in favour of nalmefene.

As-Needed Treatment with Nalmefene in

Alcohol-Dependent Patients

\section{Patient-Reported Outcomes}

At baseline, the mean SF-36 MCS score was 40.9 in both treatment groups. At week 24, the estimated mean change from baseline in the SF-36 MCS score (SE) was 2.7 (0.8) for the placebo group and 5.7 (0.8) for the nalmefene group, corresponding to a treatment difference of 3.1 (95\% CI: $1.3-4.9, \mathrm{p}=0.0008$ ) in favour of nalmefene. The mean baseline SF-36 PCS score was 50.2 and 50.8 for the placebo and nalmefene groups, respectively. At week 24, the estimated mean change from baseline in the SF-36 PCS score (SE) was $1.1(0.5)$ for the placebo group and $2.4(0.5)$ for the nalmefene group, corresponding to a treatment difference of 1.2 (95\% CI: 0.2-2.3, p = 0.0259) in favour of nalmefene.

The mean DrInC-2R score was 41.7 in the placebo group and 41.2 in the nalmefene group at baseline. At week 24, the estimated mean change from baseline in the 


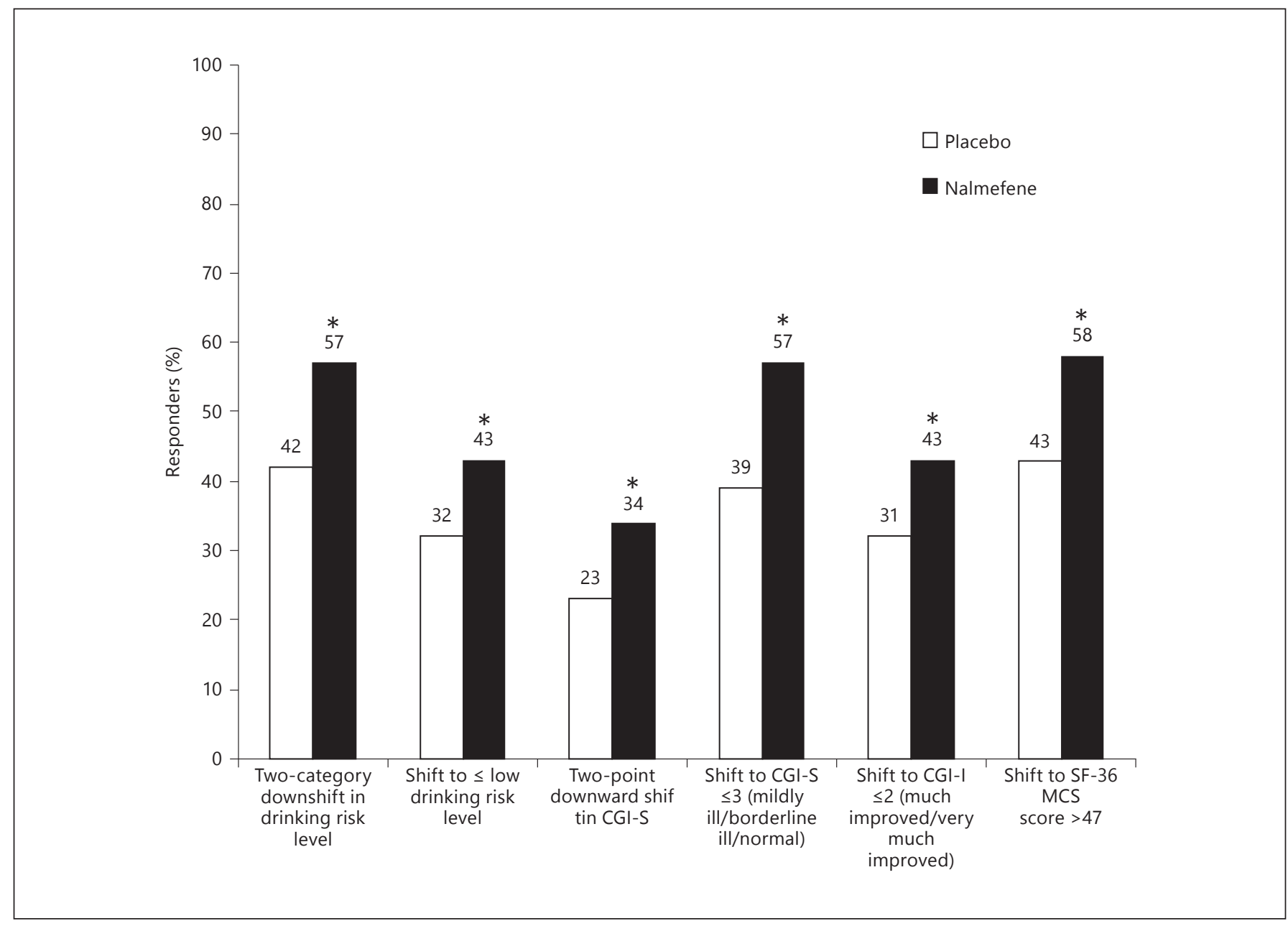

Fig. 2. Proportions of responders. * Denotes odds ratios for response in favour of nalmefene ( $p<0.05$; see table 2 for details). Missing values were imputed by response based on mixed model repeated measures predicted values.

Table 2. Odds ratios for response

\begin{tabular}{|c|c|c|c|c|c|}
\hline \multirow[t]{2}{*}{ Responder definition } & \multicolumn{2}{|c|}{$\begin{array}{l}\text { Responders at } \\
\text { month } 6 / \text { week } 24^{\text {a }}\end{array}$} & \multirow[t]{2}{*}{$\begin{array}{l}\text { Odds ratio } \\
(95 \% \mathrm{CI})\end{array}$} & \multirow[t]{2}{*}{$\mathrm{p}$} & \multirow{2}{*}{$\begin{array}{l}\text { Number- } \\
\text { needed- } \\
\text { to-treat }\end{array}$} \\
\hline & $\begin{array}{l}\text { placebo } \\
\text { responders }\end{array}$ & $\begin{array}{l}\text { nalmefene } \\
\text { responders }\end{array}$ & & & \\
\hline $\begin{array}{l}\text { Two-category downshift in drinking risk level } \\
\text { Shift to low drinking risk level (men: } \leq 40 \mathrm{~g} / \text { day; }\end{array}$ & $135(322)$ & $181(319)$ & $1.87(1.35 ; 2.59)$ & 0.0002 & 7 \\
\hline Shift to CGI-S $\leq 3$ (mildly ill/borderline ill/normal) & $125(321)$ & $182(319)$ & $2.44(1.72 ; 3.50)$ & $<0.0001$ & 6 \\
\hline $\begin{array}{l}\text { Shift to CGI-I } \leq 2 \text { (much improved/very much } \\
\text { improved) }\end{array}$ & $101(321)$ & $138(318)$ & $1.74(1.25 ; 2.44)$ & 0.0011 & 9 \\
\hline Shift to SF-36 MCS score $>47$ & $134(315)$ & $181(313)$ & $2.35(1.60 ; 3.48)$ & $<0.0001$ & 7 \\
\hline
\end{tabular}

Missing values were imputed by response based on mixed model repeated measures predicted values. ${ }^{\text {a }}$ Values in parentheses are the number of patients with baseline and post-baseline assessment of the respective response parameter in the target efficacy population. 

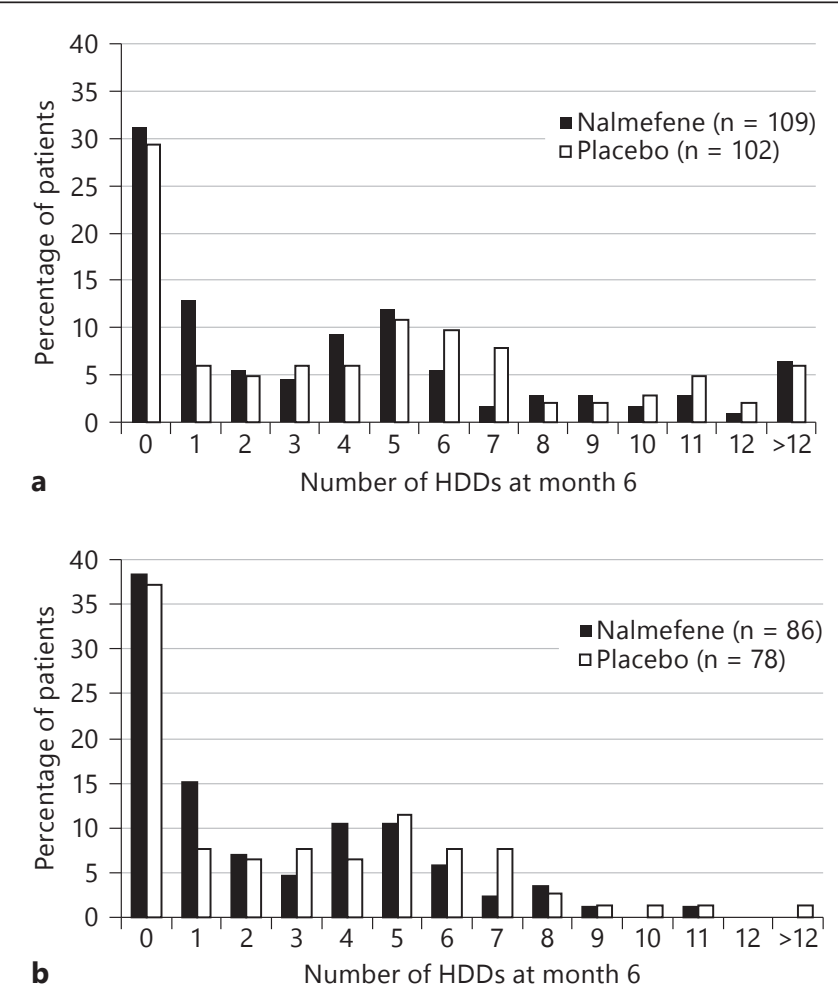

Fig. 3. Distribution of HDDs for patients defined as responders at month 6 after having a two-category downshift in drinking risk level (a) or shift to less than or equal to low drinking risk level (b) (target efficacy population, observed cases).

DrInC-2R score (SE) was -14.6 (1.3) for the placebo group and -17.9 (1.3) for the nalmefene group, corresponding to a treatment difference of -3.2 (95\% CI: -6.1 to $-0.3, p=0.0292)$ in favour of nalmefene.

\section{Liver Function Variables}

The geometric mean GGT values decreased more from baseline to week 24 in the nalmefene group than in the placebo group with a ratio of $0.82(\mathrm{p}=0.0005)$ in favour of nalmefene (table 3 ). Similar results were obtained for ALAT and ASAT with ratios of $0.85(\mathrm{p}<0.0001)$ and 0.87 $(\mathrm{p}=0.0003)$, respectively, in favour of nalmefene.

\section{Discussion}

The various responder analyses used in the current study showed a consistent pattern of clinically significant response differences between nalmefene and placebo, with odds ratios ranging from 1.79 (shift to low-risk

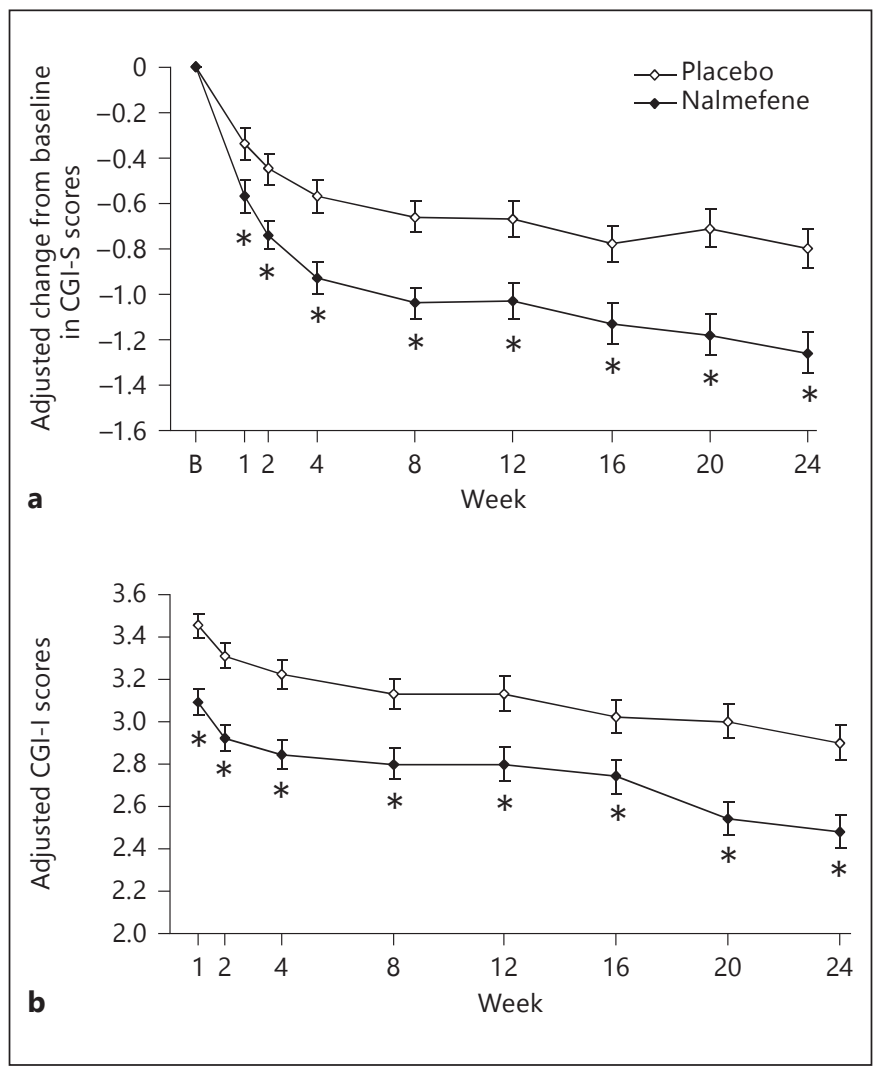

Fig. 4. Change in CGI. a Adjusted mean change from baseline in CGI-S scores. b Adjusted mean CGI-I scores. Values are means \pm SE; * $\mathrm{p}<0.05$ compared to placebo. $\mathrm{B}=$ Baseline.

drinking level) to 2.44 (shift to CGI $\leq 3$ ), and with numbers-needed-to-treat ranging from 6 to 10 . As recently described [10], nalmefene-treated patients had a mean of 3.2 fewer HDDs per month and a mean of $14.3 \mathrm{~g}$ less pure alcohol consumed per day at month 6 compared with patients on placebo; the standardised effect sizes for drinking parameters (Cohen's $\mathrm{d}=0.33$ for HDDs; 0.36 for total alcohol consumption) were larger than reported previously for licensed medications for abstinence in alcohol dependence [effect size (Hedges' g correction) of naltrexone and acamprosate on heavy drinking: $g=0.189$ and $g=0.072$, respectively] [20] and within the range for approved medicinal products in other central nervous system indications [21].

It should be noted that since nalmefene is the only drug labelled for alcohol reduction in alcohol-dependent subjects, comparison of the results in our analysis is by necessity made with drugs labelled for abstinence maintenance; in the absence of head-to-head trials, only indirect comparisons (which should be interpreted with cau- 
Table 3. Liver parameters: GGT, ALAT, and ASAT at week 24

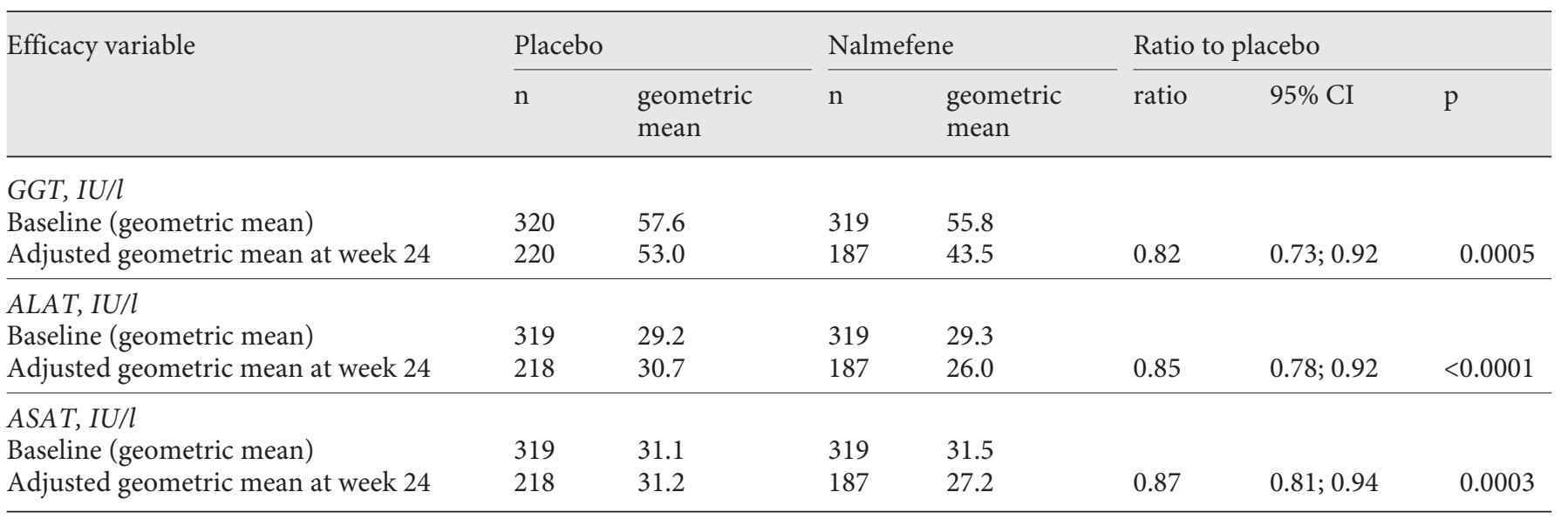

tion) can be made. While some previous trials in alcohol dependence reported marked CGI improvements [22, 23], differences between control and intervention conditions were small in size (if they existed at all [23]), The improvements in CGI that we see are comparable to those noted in studies of other mental disorders such as depression and schizophrenia $[24,25]$.

The use of the ADS and DrInC instruments as outcome measures obviously also have the merit to broaden the assessment of the impact of the intervention beyond a measurement of the drinking level. Our results show that the level of alcohol dependence, as measured by the ADS, and the severity of consequences of alcohol use, as measured by the DrInC, were both reduced with nalmefene as compared with placebo.

The improvements in the clinician-rated scales were substantiated by improvements in other patient-reported outcomes than DrInC, namely in the SF-36 MCS and PCS scores, with larger improvements in the nalmefene group (3.1 and 1.2 points, respectively) compared with placebo. These values are higher (at least for the MCS score) than those reported in an extended-release naltrexone trial aiming at reduction of alcohol consumption (improvement of 2 and 0.3 points for MCS and PCS scores compared with placebo, respectively) [26], and in a pragmatic acamprosate trial aiming at abstinence (improvement of 2.5 and 5.7 points for MCS and PCS scores compared with standard care) [22]. Importantly, it has been shown that quality of life improvements during long-term remission of alcohol dependence have the same magnitude whether or not the subjects abstain from alcohol or remit while reducing their consumption [27].
The use of biomarkers is important for the objective confirmation of patients' reported measures. The GGT, ASAT, and ALAT results confirmed the efficacy of nalmefene in reducing drinking and subsequent hepatic cytolysis in alcohol-dependent patients.

The reduction of alcohol consumption was maintained throughout the duration of the 6 -month treatment period, with no trend in any blunting of the effect with time $[2,3,10]$. This finding further supports the clinical relevance of the once controversial treatment goal of reducing consumption in alcohol-dependent patients [28], a concept which in recent years has gained increased acceptance $[6,29,30]$.

Alcohol use is the fifth leading risk factor for global disease burden [31] and reduction of drinking is the main means to reduce alcohol-attributable mortality [32]. Moreover, it has been shown that, given the exponential nature of the relationship between alcohol consumption and all-cause mortality risk, more risk is avoided by reducing the same amount of drinks from a higher level than from a lower level. For example, a reduction of $36 \mathrm{~g}$ from a baseline level of $60 \mathrm{~g}$ per day would reflect a reduced mortality risk of 38 per 10,000; however, a similar reduction of $36 \mathrm{~g}$ of alcohol per day from a baseline level of $96 \mathrm{~g}$ would reflect a reduced mortality risk of 119 per $10,000[32]$.

A meta-analysis has shown that brief intervention results in a mortality reduction of $43 \%$ [33]. Brief intervention has also been shown to reduce hospital days by $60 \%$ [34]. Since nalmefene addresses even heavier drinkers than the non-dependent heavy drinking candidates for brief intervention, it is likely that its effect on alcohol reduction would also translate into a clinically relevant re- 
duction of mortality risk in heavy-drinking alcohol-dependent subjects.

In Europe, currently fewer than $10 \%$ of subjects with an alcohol use disorder are benefiting from a treatment in a given year, a number very much lower than that found for patients with other mental disorders [35-37]. The corresponding figure for the Americas is approximately $25 \%$, which also is lower than that for all other mental disorders, except obsessive compulsive disorder [37]. It has been reported that the main reason for not seeking treatment is the reluctance to engage in abstinence [38]. In the alcohol-dependent individuals who eventually seek treatment, about $50 \%$ express a preference for a reduction goal over abstinence $[39,40]$. As nalmefene is the only drug with the treatment aim of reduced drinking, it has the potential to engage in treatment a larger number of alcohol-dependent subjects, who otherwise would have refrained from treatments offering abstinence as the sole treatment goal. Further, the as-needed regimen encourages patients to take an active part in the treatment strategy: they are invited to target their use of medication to their situations of high-risk. This procedure introduces an implicit accompanying cognitive-behavioural approach to the pharmacotherapy, and is likely to add to the clinical value of the nalmefene treatment concept.

This analysis has limitations. Firstly, the results should be interpreted in view of the fact that the study population was limited by the selection criteria, e.g. patients with active axis I co-morbidity and potentially serious withdrawal symptoms were excluded. However, this is directly in line with the European Medicines Agency Guideline on the development of medicinal products for the treatment of alcohol dependence [41]. Secondly, determining response based on drinking parameters is somewhat arbitrary as there is no single definition of what constitutes a clinically relevant reduction. However, the above-mentioned guideline states that efficacy should be evaluated in terms of responders, reflecting an expected significant improved health outcome on an individual patient level, e.g. the difference in the proportion of patients with significant categorical downshifts in the WHO drinking risk levels. Considering the baseline drinking level for these patients, a shift to a low drinking risk level is quite significant and close to or even within the drinking limits recommended by many public health authorities in many countries [42]. Finally, although the study aimed at drinking reduction, no information was collected on individual treatment goals.

As-Needed Treatment with Nalmefene in

Alcohol-Dependent Patients
In conclusion, this study demonstrates a higher proportion of treatment responders, larger improvements in patient-reported and clinician-rated outcomes, and larger improvements in liver function variables with nalmefene compared with placebo. Considering the benign safety profile of nalmefene [10], the immense alcohol-related burden to society, the harm to the individual, and the large treatment gap, the effect of nalmefene is clearly clinically relevant.

\section{Acknowledgements}

This work was funded by H. Lundbeck A/S, Valby, Denmark. We thank all patients for their participation in the studies, and all clinical research staff for their contributions. We also thank Johan Hellsten, an employee of Lundbeck, for providing medical writing assistance in the manuscript preparation, revision, and editing.

\section{Disclosure Statement}

Henri-Jean Aubin has received honoraria and travel grants from Lundbeck, Merck Serono, Ethypharm, D\&A Pharma, Pfizer, and Bioprojet. Jens Reimer is on the speaker's board of Lundbeck, Janssen-Cilag, Molteni Farmaceutici, MSD Sharp and Dohme, Sanofi-Aventis; has received honoraria (in addition to above) from Reckitt Benckiser, and is an advisor to Lundbeck, Molteni, and Reckitt Benckiser. David Nutt is a member of advisory boards for Lundbeck, Servier, Pfizer, Reckitt Benkiser, and D\&A Pharma; has received speaking honoraria (in addition to above) from BMS, GSK, Schering-Plough, Lilly; is a member of the Lundbeck International Neuroscience Foundation, and has share options in P1vital. Anna Bladström, Lars Torup, and Clément François are Lundbeck employees. Jonathan Chick has received speaker and consultancy fees from Lundbeck.

References

Eur Addict Res 2015;21:160-168

1 Bart G, Schluger JH, Borg L, Ho A, Bidlack JM, Kreek MJ: Nalmefene induced elevation in serum prolactin in normal human volunteers: partial kappa opioid agonist activity? Neuropsychopharmacology 2005;30:2254-2262.

2 Mann K, Bladström A, Torup L, Gual A, van den Brink $\mathrm{W}$ : extending the treatment options in alcohol dependence: a randomized controlled study of as-needed nalmefene. Biol Psychiatry 2013;73:706-713.

3 Gual A, He Y, Torup L, van den Brink W, Mann K: A randomised, double-blind, placebo-controlled, efficacy study of nalmefene, as-needed use, in patients with alcohol dependence. Eur Neuropsychopharm 2013;23: 1432-1442.

4 Nutt DJ: The role of the opioid system in alcohol dependence. J Psychopharmacol 2014; $28: 8-22$. 
5 World Health Organization (WHO): International Guide for Monitoring Alcohol Consumption and Related Harm. Geneva, WHO, 2000. http://whqlibdoc.who.int/ hq/2000/who_msd_msb_00.4.pdf.

6 European Medicines Agency (EMA). Find medicine. Selincro. http://www.ema.europa. eu/ema/index.jsp?curl=pages/medicines/hu$\mathrm{man} / \mathrm{medicines} / 002583 / \mathrm{human} \_\mathrm{med}$ 001620.jsp\&mid=WC0b01ac058001d124.

7 Jacobson NS, Roberts LJ, Berns SB, McGlinchey JB: Methods for defining and determining the clinical significance of treatment effects: description, application, and alternatives. J Consult Clin Psychol 1999;67:300-307.

8 European College of Neuropsychopharmacology (ECNP): Clinical relevance of response and improvement in psychopharmacology. Eur Neuropsychopharmacol 1995;5: 531-533.

9 Luquiens A, Reynaud M, Falissard B, Aubin HJ: Quality of life among alcohol-dependent patients: how satisfactory are the available instruments? A systematic review. Drug Alcohol Depend 2012;125:192-202.

10 van den Brink W, Aubin H-J, Bladström A, Torup L, Gual A, Mann K: Efficacy of asneeded nalmefene in alcohol dependent patients with at least a high drinking risk level: results from a subgroup analysis of two randomised controlled 6-month studies. Alcohol Alcohol 2013;48:570-578.

11 American Psychiatric Association: Diagnostic and Statistical Manual of Mental Disorders, ed 4, text revision (DSM-IV-TR). Washington, APA, 2000.

12 Lecrubier Y, Sheehan DV, Weiller E, Amorim P, Bonora K, Harnett Sheehan K, Janavs J, Dunbar GC: The Mini International Neuropsychiatric Interview (MINI). A short diagnostic structured interview: reliability and validity according to the CIDI. Eur Psychiatry 1997;12:224-231.

13 Starosta AN, Leeman RF, Volpicelli JR: The BRENDA Model: integrating psychosocial treatment and pharmacotherapy for the treatment of alcohol use disorders. J Psychiatr Pract 2006;12:80-89.

14 Sobell LC, Sobell MB: Timeline Follow-Back: a technique for assessing self-reported ethanol consumption; in Litten RZ, Allen JP (eds): Measuring Alcohol Consumption: Psychosocial and Biological Methods. Totowa, Humana Press, 1992, pp 41-72.

15 Guy W (ed): ECDEU Assessment Manual for Psychopharmacology. Publication No. 76338. Rockville, National Institute of Mental Health, 1976.

16 Ware JE Jr, Sherbourne CD: The MOS 36Item Short-Form Health Survey (SF-36). I. Conceptual framework and item selection. Med Care 1992;30:473-483.
17 Miller WR, Tonigan JS, Longabaugh R: The Drinker Inventory of Consequences (DrInC): An Instrument for Assessing Adverse Consequences of Alcohol Abuse. Project MATCH Monograph Series. Rockville, National Institute on Alcohol Abuse and Alcoholism, 1995.

18 Skinner HA, Horn JL: Alcohol Dependence Scale: Users Guide. Toronto, Addiction Research Foundation, 1984.

19 Maruish ME: User's manual for the SF-36v2 Health Survey, ed 3. Lincoln, QualityMetric Inc., 2011.

20 Maisel NC, Blodgett JC, Wilbourne PL, Humphreys K, Finney JW: Meta-analysis of naltrexone and acamprosate for treating alcohol use disorders: when are these medications most helpful? Addiction 2013;108:275-293.

21 Leucht S, Hierl S, Kissling W, Dold M, Davis JM: Putting the efficacy of psychiatric and general medicine medication into perspective: review of meta-analyses. Br J Psychiatry 2012;200:97-106

22 Kiritzé-Topor P, Huas D, Rosenzweig C, Comte S, Paille F, Lehert P: A pragmatic trial of acamprosate in the treatment of alcohol dependence in primary care. Alcohol Alcohol 2004;39:520-527.

23 Johnson BA, Jasinski DR, Galloway GP, Kranzler H, Weinreib R, Anton RF, Mason BJ, Bohn MJ, Pettinati HM, Rawson R, Clyde C: Ritanserin in the treatment of alcohol dependence - a multi-center clinical trial. Ritanserin Study Group. Psychopharmacology 1996; 128:206-215.

24 Lepping P, Sambhi RS, Whittington R, Lane $\mathrm{S}$, Poole R: Clinical relevance of findings in trials of antipsychotics: a systematic review. Br J Psychiatr 2011;198:341-345.

25 MacGillivray S, Arroll B, Hatcher S, Ogston S, Reid I, Sullivan F, Williams B, Crombie I: Efficacy and tolerability of selective serotonine reuptake inhibitors compared with tricyclic antidepressants in depression treated in primary care: systematic review and meta-analysis. BMJ 2003;326:1014-1017.

26 Pettinati HM, Gastfriend DR, Dong Q, Kranzler HR, O'Malley SS: Effect of extended-release naltrexone (XR-NTX) on quality of life in alcohol-dependent patients. Alcohol Clin Exp Res 2009;33:350-356.

27 Dawson DA, Li TK, Chou SP, Grant BF: Transitions in and out of alcohol use disorders: their associations with conditional changes in quality of life over a 3-year follow-up interval. Alcohol Alcohol 2009;44:84-92.

28 Sobell MB, Sobell LC: It is time for low-risk drinking goals to come out of the closet. Addiction 2011;106:1715-1717.

29 Luquiens A, Reynaud M, Aubin HJ: Is controlled drinking an acceptable goal in the treatment of alcohol dependence? A survey of French alcohol specialists. Alcohol Alcohol 2011;46:586-591.
30 van Amsterdam J, van den Brink W: Reduced-risk drinking as a viable treatment goal in problematic alcohol use and alcohol dependence. J Psychopharmacol 2013;27:987-997.

31 Lim SS, Vos T, Flaxman AD, Danaei G, Shibuya K, Adair-Rohani H, et al: A comparative risk assessment of burden of disease and injury attributable to 67 risk factors and risk factor clusters in 21 regions, 1990-2010: a systematic analysis for the Global Burden of Disease Study 2010. Lancet 2012;380:2224-2260.

32 Rehm J, Roerecke M: Reduction of drinking in problem drinkers and all-cause mortality. Alcohol Alcohol 2013;48:509-513.

33 Cuijpers P, Riper H, Lemmers L: The effects on mortality of brief interventions for problem drinking: a meta-analysis. Addiction 2004;99:839-845.

34 Kristenson $\mathrm{H}$, Ohlin $\mathrm{H}$, Hulten-Nosslin MB Trell E, Hood B: Identification and intervention of heavy drinking in middle-aged men: results and follow-up of 24-60 months of long-term study with randomized controls. Alcohol Clin Exp Res 1983;7:203-209.

35 Drummond C, Oyefeso N, Phillips T, Cheeta S, Deluca P, Perryman K, et al: Alcohol Needs Assessment Research Project (ANARP): The 2004 National Alcohol Needs Assessment for England. London, Department of Health, 2005.

36 Drummond C, Deluca P, Oyefeso A, Rome A, Scrafton S, Rice P: Scottish Alcohol Needs Assessment. London, Institute of Psychiatry, King's College London, 2009.

37 Kohn R, Saxena S, Levav I, Saraceno B: The treatment gap in mental health care. Bull World Health Organ 2013;82:858-866.

38 Substance Abuse and Mental Health Services Administration Office of Applied Studies (SAMHSA): The NSDUH Report: Alcohol Treatment: Need, Utilization, and Barriers. April 9, 2009. Rockville, SAMHSA, 2009. http://www.samhsa.gov/data/2k9/AlcTX/ AlcTX.htm.

39 Adamson SJ, Heather N, Morton V, Raistrick D; UKATT Research Team: Initial preference for drinking goal in the treatment of alcohol problems: II. Treatment outcomes. Alcohol Alcohol 2010;45:136-142.

40 Hodgins DC, Leigh G, Milne R, Gerrish R: Drinking goal selection in behavioral selfmanagement treatment of chronic alcoholics. Addict Behav 1997;22:247-255.

41 European Medicines Agency (EMA): Guideline on the development of medicinal products for the treatment of alcohol dependence. EMA/ CHMP/EWP/20097/2008. February 18, 2010. http://www.ema.europa.eu/docs/en_GB/ document_library/Scientific_guideline/ 2010/03/WC500074898.pdf.

42 Furtwaengler NA, de Visser RO: Lack of international consensus in low-risk drinking guidelines. Drug Alcohol Rev 2013;32:11-18. 